\title{
Green tea extract as a treatment for patients with wild-type transthyretin amyloidosis: an observational study
}

This article was published in the following Dove Press journal:

Drug Design, Development and Therapy

4 December 2015

Number of times this article has been viewed

\author{
Fabian aus dem Siepen' \\ Ralf Bauer' \\ Matthias Aurich' \\ Sebastian J Buss' \\ Henning Steen' \\ Klaus Altland ${ }^{2}$ \\ Hugo A Katus' \\ Arnt $\vee$ Kristen'
}

'Department of Cardiology, Angiology, and Respiratory Medicine, University

Hospital Heidelberg, Heidelberg,

Germany; ${ }^{2}$ Institute of Human

Genetics, Justus-Liebig-University,

Giessen, Germany
Correspondence: Fabian aus dem Siepen Department of Cardiology, Angiology, and Respiratory Medicine, University Hospital Heidelberg, Im Neuenheimer Feld 4I0, D-69I20 Heidelberg, Germany Tel +49622I 568611

$\mathrm{Fax}+4962215655 \mathrm{I} 5$

Email fabian.ausdemsiepen@med.uniheidelberg.de
Background: Causative treatment of patients with wild-type transthyretin amyloid cardiomyopathy (wtATTR-CM) is lacking. Recent reports indicate the potential use of epigallocatechin-3-gallate (EGCG), the most abundant catechin in green tea, to inhibit amyloid fibril formation. We sought to investigate changes of cardiac function and morphology in patients with wtATTR-CM after consumption of green tea extract (GTE).

Methods: Twenty-five male patients (71 [64; 80] years) with wtATTR-CM were submitted to clinical examination, echocardiography, cardiac magnetic resonance imaging (cMRI) $(n=14)$, and laboratory testing before and after daily consumption of GTE capsules containing $600 \mathrm{mg}$ epigallocatechin-3-gallate for at least 12 months.

Results: A significant decrease of left ventricular (LV) myocardial mass by $6 \%(196[100 ; 247]$ vs $180[85 ; 237] \mathrm{g} ; P=0.03)$ by cMRI and total cholesterol by $8.4 \%(191[118 ; 267]$ vs 173 [106; 287] $\mathrm{mg} / \mathrm{dL} ; P=0.006)$ was observed after a 1 -year period of GTE consumption. LV ejection fraction by cMRI (53\% [33\%; 69\%] vs 54\% [28\%; 71\%]; $P=0.75)$, LV wall thickness $(17[13 ; 21]$ vs $18[14 ; 25] \mathrm{mm} ; P=0.1)$, and mitral annular plane systolic excursion $(10[5 ; 23]$ vs $8[4 ; 13] \mathrm{mm} ; P=0.3$ ) by echocardiography remained unchanged.

Conclusion: This study supports LV mass stabilization in patients with wtATTR-CM consuming GTE potentially indicating amyloid fibril reduction.

Keywords: wild-type ATTR, cardiomyopathy, polyphenol, EGCG

\section{Introduction}

Transthyretin (TTR) is the most common precursor protein of hereditary amyloidosis. Its phenotype is predominantly characterized by sensorimotor polyneuropathy and/or infiltrative cardiomyopathy (CM). ${ }^{1,2}$ Moreover, TTR causes a nongenetic disease with deposition of wild-type (wt) TTR amyloid (ATTR) mainly in the heart of elderly males. ${ }^{2}$ In contrast to the hereditary form, no typical mutation in the TTR gene can be found in wtATTR patients, and the pathogenic mechanisms are a subject of ongoing research. ${ }^{3}$ Cardiac involvement is by far the most relevant predictor of outcome in both hereditary and wtATTR amyloidosis. Median survival ranged between 4 and 6 years. ${ }^{4,5}$ Cardiac wtATTR deposition was found postmortem in $25 \%$ of patients above 85 years of age. ${ }^{4} \mathrm{~A}$ recent analysis of skeletal scintigraphy revealed a prevalence of wtATTR-CM near $1.4 \%$ among males in the ninth decade of life. ${ }^{6}$

Currently, causative treatment of wtATTR-CM is lacking. Diuretics are capable of reducing dyspnea, but standard heart failure medication does not affect amyloid deposition itself. ${ }^{7}$ During the course of 1 year, wtATTR-CM usually worsens, as indicated by an increase of the left ventricular (LV) wall thickness by $0.2 \mathrm{~mm}$, an increase of 
NT-proBNP by $1,487 \mathrm{pg} / \mathrm{mL}$, and a decline of LV ejection fraction by $11 \%{ }^{8}$

Recently, in vitro experiments have shown that epigallocatechin-3-gallate (EGCG), the most abundant catechin in green tea, inhibits fibril formation of diverse amyloidogenic proteins. ${ }^{9-11}$ Disruption of ATTR fibrils was observed after a daily oral administration of $100 \mathrm{mg} / \mathrm{kg}$ EGCG for 6 weeks $^{12}$ using a transgenic mouse model of familial amyloidotic polyneuropathy carrying the human amyloidogenic Val30Met TTR variant. In a recent study, we demonstrated a decrease of LV mass in a small cohort of patients with hereditary ATTR-CM and wtATTR-CM after a daily consumption of green tea for 12 months. ${ }^{13}$ In the present study, we report on our findings in a larger, more homogenous cohort of patients with exclusively wtATTR-CM.

\section{Materials and methods}

\section{Study subjects}

Twenty-five male patients (71 [64; 80] years) were recruited at the Heidelberg Amyloidosis Center (Heidelberg, Germany) between 2008 and 2012. All patients underwent myocardial biopsy and diagnosis of wtATTR was confirmed by immunohistochemistry and molecular genetic testing. Patients started green tea consumption on their own initiative due to the rather widespread knowledge of the effects of EGCG on the disease among patients.

Two patients died during the study period, and follow-up data were not available for two further patients. Data from seven patients had already been reported in our previous study. ${ }^{13}$ All patients had stable heart failure for at least 3 months prior to study inclusion. Echocardiography, cardiac magnetic resonance imaging (cMRI) $(n=14)$, and laboratory testing, including total cholesterol, troponin-T, and NTproBNP, were performed before and after 12 months of daily consumption of 1,200 mg green tea extract (GTE) containing $600 \mathrm{mg}$ EGCG in four capsules of praevent-loges ${ }^{\circledR}$ (Dr. Loges \& $\mathrm{Co} \mathrm{GmbH}$ [Winsen, Germany]). Routine medication was continued during this period.

\section{Ethics statement}

The study was conducted according to the principles expressed in the Declaration of Helsinki and was approved by the institutional review board of the medical faculty of the University of Heidelberg, Germany (vote number S-024/2008). Written informed consent was obtained from all participants.

\section{Echocardiography}

Transthoracic echocardiograms were performed using commercially available ultrasound diagnostic systems (Vivid 7;
GE Healthcare, Milwaukee, WI, USA). Examinations were analyzed blindfolded offline on a commercially available workstation (Centricity Cardiology CA1000 2.0; GE Medical Systems, Milwaukee, WI, USA) by two experienced sonographers (MA and RB) who were unaware of all other clinical data. Thickness of interventricular septum (IVS) and posterior wall were measured in late diastole; LV cavity diameter was scaled in end diastole and end systole. Longitudinal myocardial function was quantified by mitral (MAPSE) and tricuspid (TAPSE) annular plane systolic excursion obtained from the apical four-chamber view using M-mode imaging.

\section{CMRI protocol}

All cMRI exams were performed on a 1.5 T scanner (Achieva; Philips, Best, the Netherlands) with a 32-element cardiac receiver coil and analyzed by two experienced investigators (FS and HS), both being unaware of the remaining clinical data. Vector electrocardiogram-gated standard steady-state free precession cine sequences were acquired in short axes covering the whole LV and in three long-axis views (two-, three-, and four-chamber views).

Cardiac volumes, LV ejection fraction, and myocardial mass were measured in late diastole from short-axis image stacks by manual delineation of endocardial and epicardial borders excluding papillary muscles using a commercially available workstation (Viewforum, version 3.4; Philips).

\section{Statistical analysis}

Continuous data were expressed as median (range). Categorical variables were expressed as absolute number (percent). All analyses were performed using SPSS (version 20.0, IBM Corporation, Armonk, NY, USA). Statistical significance was defined if $P$-values were $<0.05$. Differences between the nonparametric, continuous parameters assessed at study inclusion and at 12-month follow-up were compared by the Wilcoxon matched-pairs signed-rank test.

\section{Results Study cohort}

Clinical demographics of the study cohort including the subgroup of cMRI are given in Table 1. None of the patients discontinued green tea consumption and no serious side effects were reported during the study period. No changes in New York Heart Association (NYHA) class could be observed, and no significant change in surface electrocardiogram parameters (rhythm, heart rate, PQ, QRS, QTc) occurred. Two patients were lost during follow-up. One patient died due to sepsis, another due to spontaneous retroperitoneal 
Table I Baseline characteristics

\begin{tabular}{|c|c|c|c|}
\hline & $\begin{array}{l}\text { wtATTR overall } \\
(n=25)\end{array}$ & $\begin{array}{l}\text { cMRI subgroup } \\
(n=\mid 4)\end{array}$ & $P$-value \\
\hline Age (years) & $71(64 ; 80)$ & $71(64 ; 80)$ & ns \\
\hline Body weight (kg) & $80(64 ; 105)$ & $80(64 ; 105)$ & ns \\
\hline Height (cm) & $173(158 ; 190)$ & $177(168 ; 190)$ & ns \\
\hline BMI $\left(\mathrm{kg} / \mathrm{m}^{2}\right)$ & $26(20 ; 32)$ & $27(20 ; 32)$ & ns \\
\hline \multicolumn{4}{|l|}{ NYHA class } \\
\hline I & 14 (56\%) & $7(50 \%)$ & \\
\hline II & $5(20 \%)$ & $4(29 \%)$ & \\
\hline III & $6(24 \%)$ & $3(21 \%)$ & \\
\hline $\begin{array}{l}\text { Length of disease } \\
\text { (months) }\end{array}$ & 8.2 & 7.8 & ns \\
\hline \multicolumn{4}{|l|}{ ECG } \\
\hline Sinus rhythm & 21 & 12 & \\
\hline Atrial fibrillation & 4 & 2 & \\
\hline Heart rate $(\mathrm{I} / \mathrm{min})$ & $75(56 ; 91)$ & $73(56 ; 91)$ & ns \\
\hline $\mathrm{PQ}(\mathrm{ms})$ & $184(166 ; 246)$ & $184(166 ; 246)$ & ns \\
\hline QRS (ms) & II (84; I76) & $116(84 ; 170)$ & ns \\
\hline QTc (ms) & $456(410 ; 494)$ & 440 (4I0; 498) & ns \\
\hline
\end{tabular}

Note: Continuous data are expressed as median (range).

Abbreviations: cMRI, cardiac magnetic resonance imaging; ns, nonsignificant; wtATTR, wild-type transthyretin amyloidosis; BMI, body mass index; ECG, electrocardiogram; NYHA, New York Heart Association.

hematoma requiring a prolonged stay in the intensive care unit with artificial ventilation and recurrent infections, resulting in multiorgan failure and, finally, death. Follow-up over periods of 44,25 , and 30 months, respectively, was achieved in three patients.

A

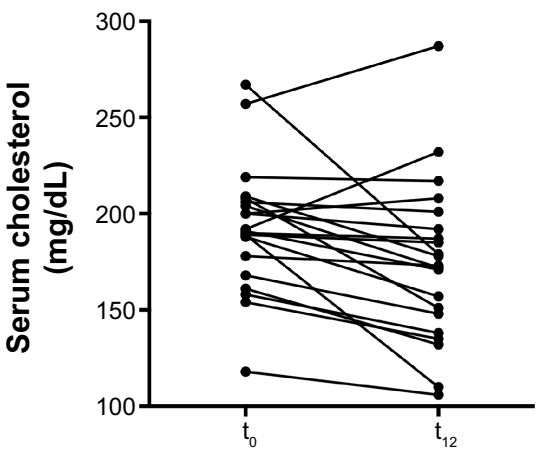

C

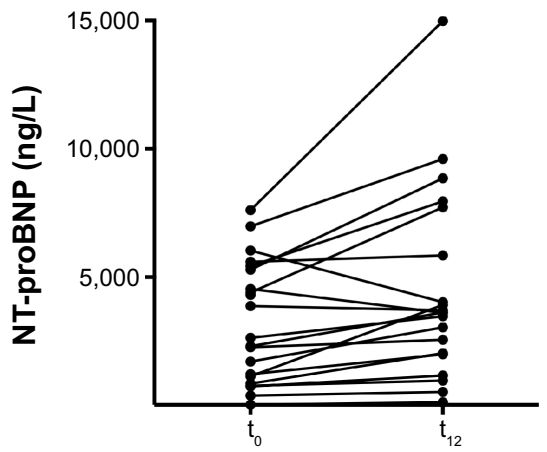

Blood analysis

In $18(85.7 \%)$ patients, a significant decrease of total serum cholesterol by $8.4 \%$ was observed (191 [118; 267] vs 173 [106; 287] $\mathrm{mg} / \mathrm{dL} ; P=0.006)$ during the 12 -month study period. In three patients, an increase of cholesterol by $3 \%$ was observed. In $16(76.2 \%)$ patients, low-density lipoprotein (LDL) cholesterol decreased by 8.0\% (110 [60; 180] vs 99 $[55 ; 188] \mathrm{mg} / \mathrm{dL} ; P=0.02])$. In four patients, LDL cholesterol increased by $7 \%$.

Median NT-proBNP levels remained stable (3,681 vs $3,617 \mathrm{ng} / \mathrm{L}$ ) in the whole study group during the observation period. Glomerular filtration rate calculated by the modified diet in renal disease (MDRD) formula (62 [31;99] vs 57 [26; 101] $\mathrm{mL} / \mathrm{min} ; P=0.23)$, GOT $(30[16 ; 56]$ vs 33 [17; 74] U/L; $P=0.90)$, and GPT $(29[10 ; 57]$ vs 29 [10; 74] U/L; $P=0.95)$ remained unchanged. TTR plasma levels remained stable during the study period $(0.28[0.14 ; 0.90]$ vs 0.26 [0.18; 0.50$] \mathrm{mg} / \mathrm{dL} ; P=0.64 ; \mathrm{n}=16)$. Results of laboratory testing are shown in Figure 1 and Table 2.

\section{Echocardiography analysis}

Mean thickness of IVS $(17[13 ; 28]$ vs 18 [14;25] $\mathrm{mm} ; P=0.1)$ and posterior wall $(16[11 ; 24]$ vs 17 $[12 ; 19] \mathrm{mm} ; P=0.4)$ remained unchanged during the study period (Figure 2).
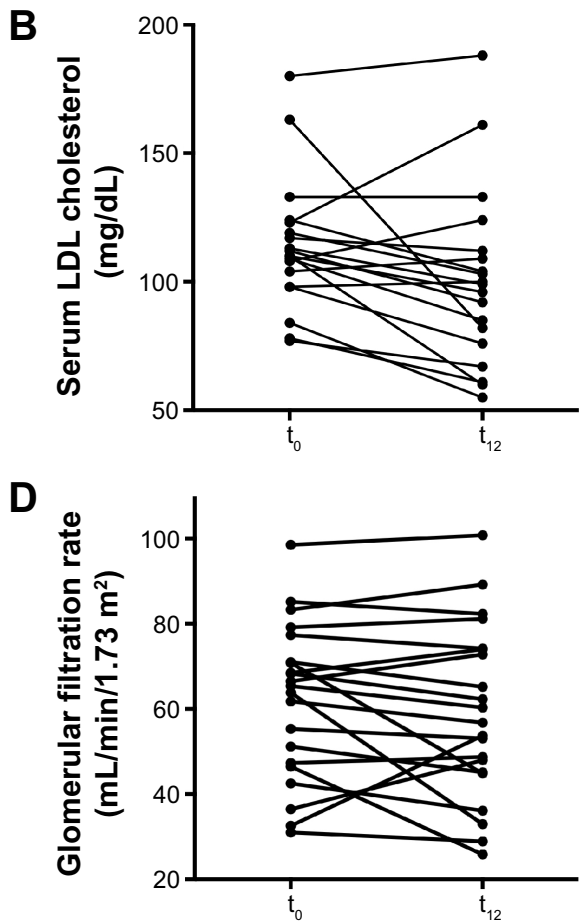

Figure I Laboratory testing.

Notes: Levels of cholesterol (A), LDL cholesterol (B), NT-proBNP (C), and glomerular filtration rate calculated using the MDRD formula (D) of individual patients with wtATTR-CM determined by cMRI before and after 12 months of green tea extract consumption.

Abbreviations: cMRI, cardiac magnetic resonance imaging; LDL, low-density lipoprotein; MDRD, modified diet in renal disease; $t_{0}$, baseline; $t_{12}$, at 12 months; wtATTR-CM, wild-type transthyretin amyloid cardiomyopathy; NT-proBNP, N-terminal prohormone of brain natriuretic peptide. 
Table 2 Blood analysis

\begin{tabular}{|c|c|c|c|}
\hline & $\begin{array}{l}\text { Study inclusion } \\
(\mathrm{n}=25)\end{array}$ & $\begin{array}{l}\text { I } 2 \text { months after } \\
\text { study start }(n=21)\end{array}$ & $P$-value \\
\hline Creatinine (mg/dL) & $1.2(0.8 \mathrm{I} ; 2.3)$ & I.4 $(0.79 ; 2.6)$ & ns \\
\hline $\operatorname{MDRD}(\mathrm{mL} / \mathrm{min})$ & $62(31 ; 99)$ & $57(26 ; 10 \mathrm{I})$ & ns \\
\hline GOT (U/L) & $30(16 ; 56)$ & $33(17 ; 74)$ & ns \\
\hline GPT (U/L) & $29(10 ; 57)$ & $29(10 ; 74)$ & ns \\
\hline Cholesterol (mg/dL) & $191(118 ; 267)$ & I73 (106; 287) & 0.006 \\
\hline $\mathrm{HDL}(\mathrm{mg} / \mathrm{dL})$ & $48(30 ; 78)$ & $46(31 ; 75)$ & ns \\
\hline $\mathrm{LDL}(\mathrm{mg} / \mathrm{dL})$ & $124(60 ; 180)$ & $99(55 ; 188)$ & 0.02 \\
\hline
\end{tabular}

Note: Continuous data are expressed as median (range).

Abbreviations: GOT, glutamic-oxaloacetic transaminase; GPT, glutamic-pyruvic transaminase; HDL, high-density lipoprotein; LDL, low-density lipoprotein; MDRD, modified diet in renal disease; ns, nonsignificant.

In seven (33.3\%) patients, an increase of either 1) both IVS and posterior wall thickness $(\mathrm{n}=4)$ or 2$)$ isolated posterior wall thickness or IVS thickness ( $\mathrm{n}=3$ ) occurred, by an average of $1.9 \mathrm{~mm}(13 \%)$.

MAPSE $(10[5 ; 23]$ vs $8[4 ; 13] \mathrm{mm} ; P=0.3)$, TAPSE $(14[8 ; 23]$ vs $15[2 ; 20] \mathrm{mm} ; P=0.4)$, and passive mitral inflow/lateral early diastolic mitral annular velocity (E/E') ratio $(11[7 ; 28]$ vs $14[4 ; 31] ; P=0.8)$ remained unchanged. Results of echocardiography are shown in Table 3 .

\section{cMRI analysis}

In the subgroup of patients assessed by cMRI $(n=14)$ (Figure 3; Table 4), in 12 (86\%) patients, a decrease of LV myocardial mass by $5.9 \%$ was observed during follow-up $(196[100 ; 247]$ vs $180[85 ; 237] \mathrm{g} ; P=0.03)$. In the remaining two patients, an increase by $11 \%$ and by $15 \%$ was observed, respectively.

LV ejection fraction (53\% [33\%; 69\%] vs 54\% [28\%; $71 \%] ; P=0.75$ ) and longitudinal function (MAPSE: 6.5
Table 3 Echocardiography

\begin{tabular}{|c|c|c|c|}
\hline & $\begin{array}{l}\text { Study inclusion } \\
(n=25)\end{array}$ & $\begin{array}{l}12 \text { months after } \\
\text { study start }(n=21)\end{array}$ & $P$-value \\
\hline IVS thickness (mm) & $17(13 ; 28)$ & $18(14 ; 25)$ & ns \\
\hline $\begin{array}{l}\text { LV Inferior wall } \\
\text { thickness }(\mathrm{mm})\end{array}$ & $16(11 ; 24)$ & $17(12 ; 19)$ & ns \\
\hline LV myocardial mass $(\mathrm{g})$ & $340(173 ; 523)$ & $335(228 ; 50 I)$ & ns \\
\hline LVEDD (mm) & $44(36 ; 53)$ & $42(33 ; 54)$ & ns \\
\hline LVESD (mm) & $33(23 ; 45)$ & $32(24 ; 49)$ & ns \\
\hline MAPSE (mm) & $10(5 ; 23)$ & $8(4 ; 13)$ & ns \\
\hline TAPSE (mm) & $14(8 ; 23)$ & $15(2 ; 20)$ & ns \\
\hline$E / E '$ & II $(7 ; 28)$ & $14(4 ; 31)$ & ns \\
\hline
\end{tabular}

Note: Continuous data are expressed as median (range).

Abbreviations: E/E', passive mitral inflow/lateral early diastolic mitral annular velocity; IVS, interventricular septum; LVEDD, left ventricular end-diastolic diameter; LVESD, left ventricular end-systolic diameter; MAPSE, mitral annular plane systolic excursion; ns, nonsignificant; TAPSE, tricuspid annular plane systolic excursion.

$[3 ; 10]$ vs $6[3 ; 9] \mathrm{mm} ; P=0.08$ and TAPSE: $12[4 ; 17]$ vs 11 $[5 ; 16] \mathrm{mm} ; P=0.28)$ remained unchanged.

LV mass of the three patients with long-term observation are shown in Figure 4. Patient 1 showed a reduction of $11 \mathrm{~g}$ (4.4\%) after 44 months, patient 2 an increase of $5 \mathrm{~g}(3.2 \%)$ after 25 months, and patient 3 a reduction of $10 \mathrm{~g}(10 \%)$ after 30 months.

\section{Discussion}

We report on a cohort of wtATTR-CM patients consuming GTE for at least 1 year to demonstrate potential effects on inhibition of amyloid fibril formation. ${ }^{10,14}$ A significant decrease of LV myocardial mass was observed by cMRI while no change of LV wall thickness was detected by echocardiography. A decrease of LV mass was found in two of three patients after 30 and 44 months of observation, while there was an increase by $3 \%$ in the third patient after 25 months of treatment. No side effects were reported during the study period. Within a time span of 1 year, ATTR-CM
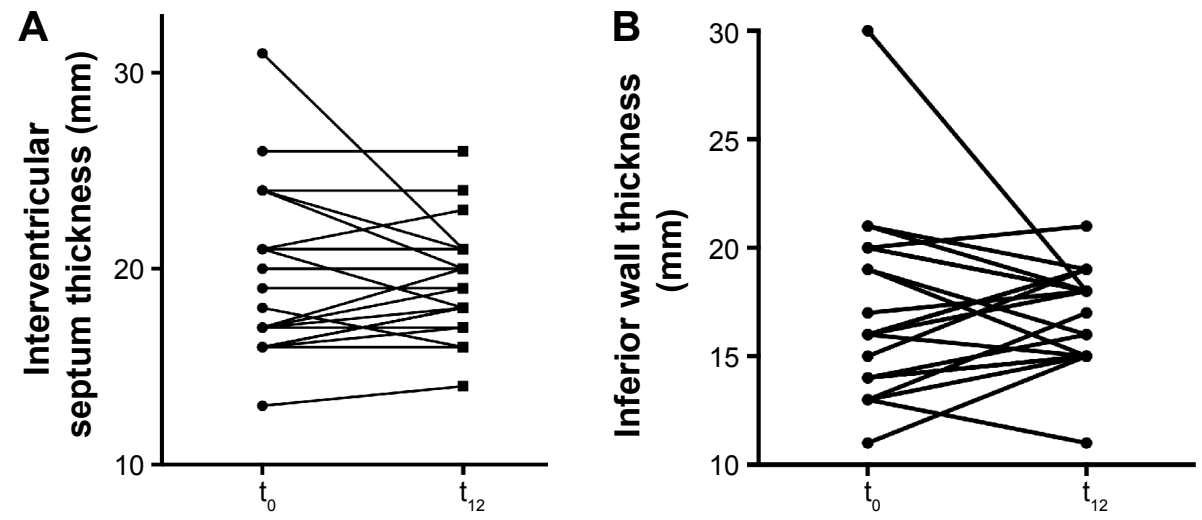

Figure 2 Echocardiography findings.

Notes: Interventricular septum thickness (A) and inferior wall thickness (B) of individual patients with wtATTR-CM determined by echocardiography before and after 12 months of green tea extract consumption.

Abbreviations: $\mathrm{t}_{0}$, baseline; $\mathrm{t}_{12}$, at 12 months; wtATTR-CM, wild-type transthyretin amyloid cardiomyopathy. 

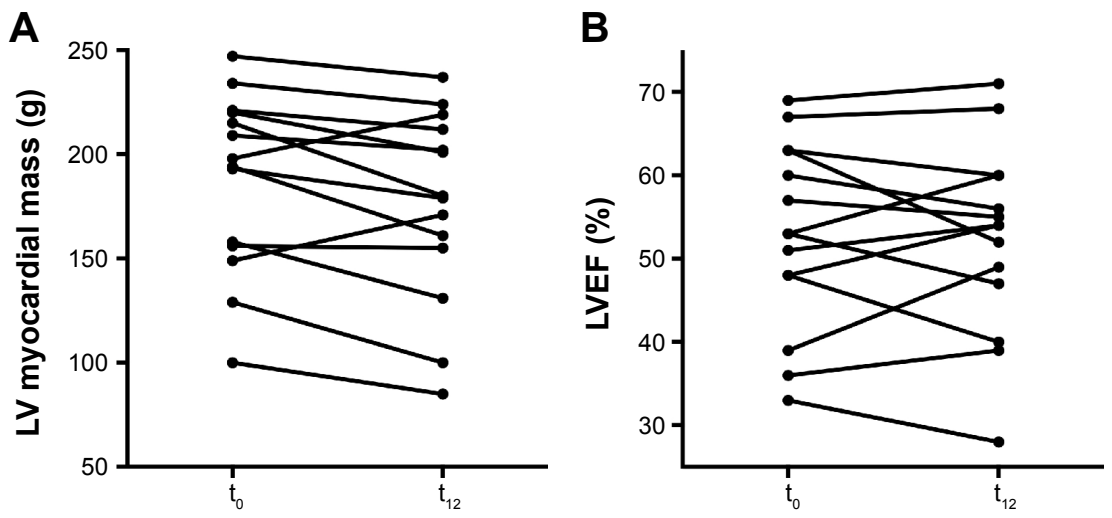

Figure 3 cMRI findings.

Notes: LV myocardial mass (A) and LVEF (B) of individual patients with wtATTR-CM determined by cMRI before and after I2 months of green tea extract consumption. Abbreviations: cMRI, cardiac magnetic resonance imaging; LV, left ventricular; LVEF, LV ejection fraction; $t_{0}$, baseline; $t_{12}$, at I2 months; wtATTR-CM, wild-type transthyretin amyloid cardiomyopathy.

was found to be associated with a significant increase of LV myocardial mass and IVS thickness as well as a reduction of LV ejection fraction. ${ }^{8,15}$ Thus, we concluded that EGCG may halt progression of this fatal disease.

wtATTR-CM is characterized by predominantly cardiac deposition of wtATTR. According to more recent data achieved by skeletal scintigraphy, it appears to cause LV infiltration in up to $1 \%$ of patients above 60 years of age. ${ }^{6}$ Without treatment, ATTR-CM was found to be associated with an increase of LV mass by $8 \%$ within a 1 -year period $^{15}$ and a decrease of LV ejection fraction by $3.2 \%$ during 6 months. ${ }^{8}$ An association between amyloid load and myocardial mass could be demonstrated in a mouse model by Ogawa. ${ }^{16}$ Causative treatment options are lacking. In other studies, prognosis of patients with ATTR-CM remained poor, with median survival of 4-6 years. ${ }^{5,17}$ Diverse in vitro and in vivo strategies have been aimed to stop amyloid fibril formation by stabilization of TTR protein (tafamidis [Vyndaqel $\left.{ }^{\circledR}\right]$, EGCG) and by RNA interference using ALNTTRsc (Revusiran ${ }^{\circledR}$ ) or ALN-TTR02 (Patisiran ${ }^{\circledR}$ ) or to dissolve amyloid fibrils by EGCG. The health benefit of green

Table 4 Cardiac magnetic resonance imaging

\begin{tabular}{|c|c|c|c|}
\hline & $\begin{array}{l}\text { Study inclusion } \\
(n=14)\end{array}$ & $\begin{array}{l}12 \text { months after } \\
\text { study start }(n=14)\end{array}$ & $P$-value \\
\hline LVEF (\%) & $53(33 ; 69)$ & $54(28 ; 7 I)$ & ns \\
\hline EDV $(m L)$ & 161 (87; 278) & $162(102 ; 289)$ & ns \\
\hline ESV $(m L)$ & $69(40 ; 185)$ & $76(40 ; 207)$ & ns \\
\hline LV myocardial mass (g) & $196(100 ; 247)$ & $180(85 ; 237)$ & 0.03 \\
\hline MAPSE (mm) & $7(3 ; 10)$ & $6(3 ; 9)$ & ns \\
\hline TAPSE (mm) & $12(4 ; 17)$ & II $(5 ; 16)$ & ns \\
\hline
\end{tabular}

Note: Continuous data are expressed as median (range).

Abbreviations: EDV, end-diastolic volume; ESV, end-systolic volume; LVEF, left ventricular ejection fraction; MAPSE, mitral annular plane systolic excursion; ns, nonsignificant; TAPSE, tricuspid annular plane systolic excursion. tea, in particular EGCG, has been reported for many years, ${ }^{18}$ and only rarely were adverse effects ascribed to the consumption of GTE. ${ }^{19}$ Actually, two clinical Phase III studies using tafamidis and RNA interference drugs are recruiting patients, but high costs and lack of long-term data on toxicity have to be considered as limitations so far. ${ }^{20}$

Green tea is considered to positively affect cardiovascular and metabolic complications ${ }^{21}$ and to improve lipid profile. ${ }^{12}$ Thus, decrease of LDL cholesterol appears to be an indicator of GTE bioavailability. To assure a daily constant intake dose of EGCG, and to facilitate fluid restriction in those patients with severe heart failure, we administered capsules containing EGCG and vitamin C. Vitamin C improves the intestinal stability of EGCG. ${ }^{22,23}$

Hunstein's experiment to treat his light-chain amyloidosis with green tea ${ }^{14}$ was stimulated by the experiments of Bieschke J et $\mathrm{al}^{9}$ and Ehrnhoefer DE et al, ${ }^{10}$ who demonstrated amyloid fibril disruption incubated with EGCG. In a retrospective analysis of a larger cohort of patients with light-chain amyloidosis, a significant reduction of myocardial mass and an improvement of LV ejection fraction and NYHA functional class were reported in patients consuming green tea, while no changes were observed in patients without intake of green tea. ${ }^{24}$

Recently, we demonstrated by cMRI an average reduction of LV myocardial mass by $12.5 \%$ in a heterogeneous cohort of patients with mutant ATTR $(n=8)$ and wtATTR $(n=6) .{ }^{13}$ This study was focused on patients with wtATTR-CM to avoid modifying effects from inherited forms of ATTR-CM. It remains unclear why some patients did not respond to GTE. Different individual metabolic conditions and renal excretion might have an impact on EGCG plasma concentration. Up to now, no standardized test has been validated that allows reproducible quantification of EGCG plasma concentration. 

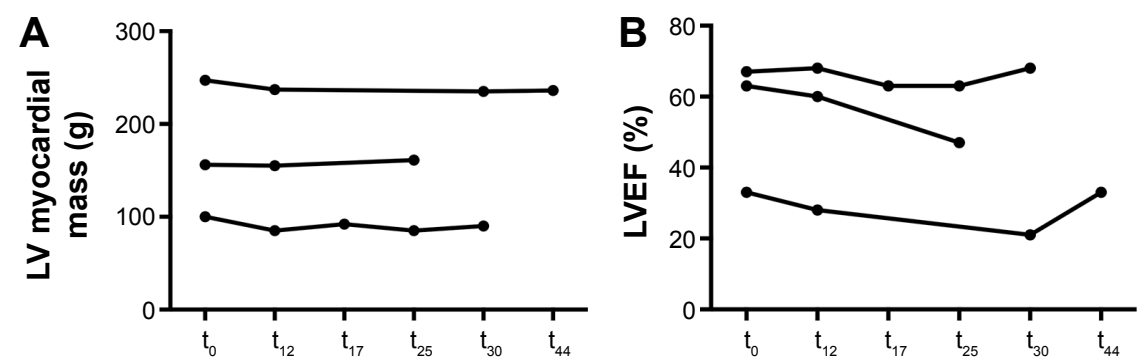

Figure 4 LV myocardial mass (A) and LVEF (B) of patients with wtATTR-CM who underwent assessment by cMRI with green tea extract consumption for longer than I year. Abbreviations: $c M R I$, cardiac magnetic resonance imaging; LV, left ventricular; LVEF, LV ejection fraction; $t_{0}$, baseline; $t_{12}$, at 12 months; $t_{17}$, at 17 months; $t_{25}$, at 25 months; $\mathrm{t}_{30}$, at 30 months; $\mathrm{t}_{44}$, at 44 months; wtATTR-CM, wild-type transthyretin amyloid cardiomyopathy.

We observed a disagreement between echocardiography and cMRI data. We assumed that the higher inter- and intraobserver variability of echocardiography within a small sample size could be a reasonable explanation. ${ }^{25} \mathrm{cMRI}$ is considered as the gold standard for analysis of cardiac morphology and function. ${ }^{26,27}$

Compared with other types of cardiac amyloidosis, wtATTR-CM progresses slowly. Demonstration of a longterm benefit of EGCG requires a longer follow-up beyond 1 year. According to the mechanism of action, it is proposed that EGCG has an additional effect on amyloid fibril formation and disruption to novel drugs that have been reported to inhibit fibril formation from TTR. Tafamidis, a novel stabilizer of human TTR, ${ }^{28}$ has been shown to stop progression of ATTR V30M polyneuropathy in two-thirds of patients..$^{29,30}$ It was approved by the European Medicines Agency in December 2011 and by the Japanese Pharmaceuticals and Medical Devices Agency in September 2013 for treatment of stage 1 polyneuropathy in patients with hereditary ATTR amyloidosis. ATTR-ACT, a Phase III study on the impact of tafamidis on cardiac ATTR amyloidosis, started in December 2013. ${ }^{31}$ The well-known different binding sites of tafamidis and EGCG suggest a synergistic effect.

This is an observational report on a relatively small, but well-sized, study cohort with respect to the prevalence of this disease. As green tea is widely available, of low cost, and, importantly, appears to have only minor side effects with long-term use, the potential for a placebo-controlled clinical trial is limited. Furthermore, EGCG capsules are over-the-counter products, and the broad spectrum of effects of EGCG, including amyloid fibril inhibition, has been well known for many years to most ATTR amyloidosis patients. Thus, we were not able to recruit a control group for the present study. None of the fatal events appeared to be associated with intake of GTE.

We assumed that the observed reduction of myocardial mass was due to reduction of amyloid load. Nevertheless, cell atrophy or hypotrophy might explain these findings as well. Further MRI studies demonstrated the use of a novel technique for tissue characterization, T1 mapping, for early diagnosis ${ }^{32-34}$ and prognosis ${ }^{35}$ in different forms of cardiac amyloidosis. T1 mapping allows quantification of the extracellular volume fraction. Recently, reduction of extracellular volume by T1 mapping indicative of reduction of amyloid load has been demonstrated in a small cohort of patients with ATTR amyloidosis. ${ }^{36}$ However, histopathological validation of this technique is lacking.

\section{Conclusion}

This study supports evidence for the benefit of EGCG to stabilize myocardial mass in wtATTR-CM patients. A reduction of myocardial mass was observed by cMRI, while echocardiographic data and cardiac biomarkers, however, remained unchanged during the study period of 1 year.

\section{Acknowledgment}

This study was financially supported by Dr. Loges \& Co $\mathrm{GmbH}$ (Winsen, Germany) by providing the green tea capsules for the patients.

\section{Disclosure}

The authors report no conflicts of interest in this work. No payments were received. Dr. Loges \& $\mathrm{Co} \mathrm{GmbH}$ (Winsen, Germany) provided capsules of green tea extract and support to conduct the study, but were not involved in the study design, the analysis, or the interpretation of the results.

\section{References}

1. Connors LH, Doros G, Sam F, Badiee A, Seldin DC, Skinner M. Clinical features and survival in senile systemic amyloidosis: comparison to familial transthyretin cardiomyopathy. Amyloid. 2011;18 Suppl 1:157-159.

2. Pomerance A. Senile cardiac amyloidosis. Br Heart J. 1965;27(5): 711-718

3. Polimanti R, Di Girolamo M, Manfellotto D, Fuciarelli M. In silico analysis of TTR gene (coding and non-coding regions, and interactive network) and its implications in transthyretin-related amyloidosis. Amyloid. 2014;21(3):154-162. 
4. Cornwell GG 3rd, Murdoch WL, Kyle RA, Westermark P, Pitkänen P. Frequency and distribution of senile cardiovascular amyloid. A clinicopathologic correlation. Am J Med. 1983;75(4):618-623.

5. Pinney JH, Whelan CJ, Petrie A, et al. Senile systemic amyloidosis: clinical features at presentation and outcome. J Am Heart Assoc. 2013; 2(2): $\mathrm{e} 000098$.

6. Longhi S, Guidalotti PL, Quarta CC, et al. Identification of TTR-related subclinical amyloidosis with 99mTc-DPD scintigraphy. JACC Cardiovasc Imaging. 2014;7(5):531-532.

7. Mohty D, Damy T, Cosnay P, et al. Cardiac amyloidosis: updates in diagnosis and management. Arch Cardiovasc Dis. 2013;106(10):528-540.

8. Ruberg FL, Maurer MS, Judge DP, et al. Prospective evaluation of the morbidity and mortality of wild-type and V122I mutant transthyretin amyloid cardiomyopathy: the Transthyretin Amyloidosis Cardiac Study (TRACS). Am Heart J. 2012;164(2):222-228.e1.

9. Bieschke J, Russ J, Friedrich RP, et al. EGCG remodels mature alpha-synuclein and amyloid-beta fibrils and reduces cellular toxicity. Proc Natl Acad Sci U S A. 2010;107(17):7710-7715.

10. Ehrnhoefer DE, Bieschke J, Boeddrich A, et al. EGCG redirects amyloidogenic polypeptides into unstructured, off-pathway oligomers. Nat Struct Mol Biol. 2008;15(6):558-566.

11. Ferreira N, Cardoso I, Domingues MR, et al. Binding of epigallocatechin3-gallate to transthyretin modulates its amyloidogenicity. FEBS Lett. 2009;583(22):3569-3576.

12. Ferreira N, Saraiva MJ, Almeida MR. Epigallocatechin-3-gallate as a potential therapeutic drug for TTR-related amyloidosis: "in vivo" evidence from FAP mice models. PLoS One. 2012;7(1):e29933.

13. Kristen AV, Lehrke S, Buss S, et al. Green tea halts progression of cardiac transthyretin amyloidosis: an observational report. Clin Res Cardiol. 2012;101(10):805-813.

14. Hunstein W. Epigallocathechin-3-gallate in AL amyloidosis: a new therapeutic option? Blood. 2007;110(6):2216.

15. Benson MD, Teague SD, Kovacs R, Feigenbaum H, Jung J, Kincaid JC. Rate of progression of transthyretin amyloidosis. Am J Cardiol. 2011; 108(2):285-289.

16. Ogawa $\mathrm{H}$. Senile cardiac amyloidosis in senescence accelerated mouse (SAM). Jpn Circ J. 1988;52(12):1377-1383.

17. Rapezzi C, Merlini G, Quarta CC, et al. Systemic cardiac amyloidoses: disease profiles and clinical courses of the 3 main types. Circulation. 2009;120(13):1203-1212.

18. Khurana S, Venkataraman K, Hollingsworth A, Piche M, Tai TC. Polyphenols: benefits to the cardiovascular system in health and in aging. Nutrients. 2013;5(10):3779-3827.

19. Sarma DN, Barrett ML, Chavez ML, et al. Safety of green tea extracts: a systematic review by the US Pharmacopeia. Drug Saf. 2008;31(6): 469-484.

20. Obici L, Merlini G. An overview of drugs currently under investigation for the treatment of transthyretin-related hereditary amyloidosis. Expert Opin Investig Drugs. 2014;23(9):1239-1251.

21. Keske MA, Ng HL, Premilovac D, et al. Vascular and metabolic actions of the green tea polyphenol epigallocatechin gallate. Curr Med Chem. 2015;22(1):59-69.
22. Chung JH, Kim S, Lee SJ, Chung JO, Oh YJ, Shim SM. Green tea formulations with vitamin $\mathrm{C}$ and xylitol on enhanced intestinal transport of green tea catechins. J Food Sci. 2013;78(5):C685-C690.

23. Peters CM, Green RJ, Janle EM, Ferruzzi MG. Formulation with ascorbic acid and sucrose modulates catechin bioavailability from green tea. Food Res Int. 2010;43(1):95-102.

24. Mereles D, Buss SJ, Hardt SE, Hunstein W, Katus HA. Effects of the main green tea polyphenol epigallocatechin-3-gallate on cardiac involvement in patients with AL amyloidosis. Clin Res Cardiol. 2010; 99(8):483-490.

25. Perdrix L, Mansencal N, Cocheteux B, et al. How to calculate left ventricular mass in routine practice? An echocardiographic versus cardiac magnetic resonance study. Arch Cardiovasc Dis. 2011;104(5): 343-351.

26. Bellenger NG, Burgess MI, Ray SG, et al. Comparison of left ventricular ejection fraction and volumes in heart failure by echocardiography, radionuclide ventriculography and cardiovascular magnetic resonance; are they interchangeable? Eur Heart J. 2000;21(16):1387-1396.

27. Bellenger NG, Davies LC, Francis JM, Coats AJ, Pennell DJ. Reduction in sample size for studies of remodeling in heart failure by the use of cardiovascular magnetic resonance. J Cardiovasc Magn Reson. 2000;2(4):271-278.

28. Bulawa CE, Connelly S, Devit M, et al. Tafamidis, a potent and selective transthyretin kinetic stabilizer that inhibits the amyloid cascade. Proc Natl Acad Sci U S A. 2012;109(24):9629-9634.

29. Coelho T, Maia LF, Martins da Silva A, et al. Tafamidis for transthyretin familial amyloid polyneuropathy: a randomized, controlled trial. Neurology. 2012;79(8):785-792.

30. Merkies IS. Tafamidis for transthyretin familial amyloid polyneuropathy: a randomized, controlled trial. Neurology. 2013;80(15):1444-1445.

31. Ueda M, Ando Y. Recent advances in transthyretin amyloidosis therapy. Transl Neurodegener. 2014;3:19.

32. Brooks J, Kramer CM, Salerno M. Markedly increased volume of distribution of gadolinium in cardiac amyloidosis demonstrated by T1 mapping. J Magn Reson Imaging. 2013;38(6):1591-1595.

33. Fontana M, Banypersad SM, Treibel TA, et al. Native T1 mapping in transthyretin amyloidosis. JACC Cardiovasc Imaging. 2014;7(2): 157-165.

34. Karamitsos TD, Piechnik SK, Banypersad SM, et al. Noncontrast T1 mapping for the diagnosis of cardiac amyloidosis. JACC Cardiovasc Imaging. 2013;6(4):488-497.

35. Maceira AM, Prasad SK, Hawkins PN, Roughton M, Pennell DJ. Cardiovascular magnetic resonance and prognosis in cardiac amyloidosis. J Cardiovasc Magn Reson. 2008;10:54.

36. aus dem Siepen F, Buss SJ, Andre F, et al. Extracellular remodeling in patients with wild-type amyloidosis consuming epigallocatechin-3gallate: preliminary results of T1 mapping by cardiac magnetic resonance imaging in a small single center study. Clin Res Cardiol. 2015; 104(8):640-647.

\section{Publish your work in this journal}

Drug Design, Development and Therapy is an international, peerreviewed open-access journal that spans the spectrum of drug design and development through to clinical applications. Clinical outcomes, patient safety, and programs for the development and effective, safe, and sustained use of medicines are a feature of the journal, which

\section{Dovepress}

has also been accepted for indexing on PubMed Central. The manuscript management system is completely online and includes a very quick and fair peer-review system, which is all easy to use. Visit http://www.dovepress.com/testimonials.php to read real quotes from published authors. 